\title{
A social robot mediator to foster collaboration and inclusion among children
}

\author{
Sarah Gillet \\ KTH, Royal Institute of Technology \\ sgillet@kth.se
}

\author{
Wouter van den Bos \\ University of Amsterdam \\ w.vandenBos@uva.nl
}

\author{
Iolanda Leite \\ KTH, Royal Institute of Technology \\ iolanda@kth.se
}

\begin{abstract}
Formation of subgroups and thereby the problem of intergroup bias is well-studied in psychology. Already from the age of five, children can show ingroup preferences. We developed a social robot mediator to explore how a robot could help overcome these intergroup biases, especially for children newly arrived to a country. By utilizing an online evaluation of collaboration levels, we allow the robot to perceive and act upon the current group dynamics. We investigated the effectiveness of the robot's mediating behavior in a between-subject study with 39 children, of whom 13 children had arrived in Sweden within the last 2 years. Results indicate that the robot could help the process of inclusion by mediating the activity. The robot succeeds in encouraging the newly arrived children to act more outgoing and in increasing collaboration among ingroup children. Further, children show a higher level of prosociality after interacting with the robot. In line with prior work, this study demonstrates the ability of social robotic technology to assist group processes.
\end{abstract}

\section{INTRODUCTION}

Today's society is increasingly polarized with growing impressions of "us vs. them" in various aspects of everyday life. A persistent challenge is the discrimination between ingroup and outgroup members which has been extensively studied in psychology. Research has shown that people tend to act more favorably towards ingroup members than to outgroup members [5, 19]. From an age as young as 5, children can have ingroup preferences, i.e., they exhibit favoritism of ingroup members over outgroup members in their evaluation of others or allocation of resources [2].

At the same time, the world is becoming increasingly diverse and multicultural. The World Migration Report 2020 [1] reports an increasing number of people that live in another country than their country of birth (3.5\% of the world population, 272 million people) and $14 \%$ are younger than 20 years. Consequently, more children that newly arrive to a country have to face the challenge of cultural adaptation in addition to the challenge of inclusion in the new environment.

Literature in psychology shows how social relationships among children could be improved through collaborative learning [11] and group interaction [7], and how joint music making can enhance prosociality and cooperation [10]. Moreover, advances in the field of human-robot interaction have shown that robots can influence group dynamics such as group cohesion [15], conflict solving strategies [9] or balanced participation [20]. Recent advances also showed how robots could improve team inclusion among adults [18].

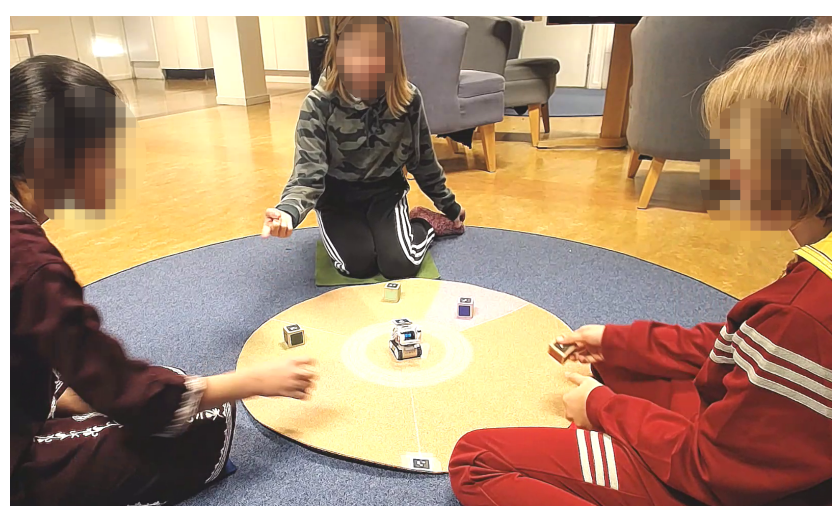

Fig. 1: Children playing the music-based puzzle with Cozmo mediating the play to foster collaboration and inclusion.

In this work, our goal is to use a robotic mediator to facilitate inclusion of outgroup members in the ingroup while fostering spontaneous collaboration. We developed a musicbased puzzle built around the placement of tangible elements. By observing the children's behavior in the puzzle, we show that the robot can evaluate collaboration and inclusion tendencies online and act upon these (see Fig. 11). To promote collaboration and inclusion, the robot encourages the players to equally explore different placements of the tangible elements. This, for example, results in the robot suggesting placements in areas that intuitively belong to other players.

We study the effectiveness of the robot mediation in combination with a special target group, children that newly arrived in a country. These children (potentially outgroup members) are paired with children already present in the new school environment to build a group of three.

\section{RELATED WORK}

Robots can shape group dynamics in different ways and for a range of group settings. First, robots have shown to attribute roles and facilitate intimacy in interactions. A pioneering study by Mutlu et al. [12] showed that a robot's gaze can influence people's conversational roles. Another study showed that robots can increase engagement and trust-related behaviors between team members who are making vulnerable statements [17]. A robotic therapist has been shown to improve intimacy and positive affect between romantic couples [21]. 


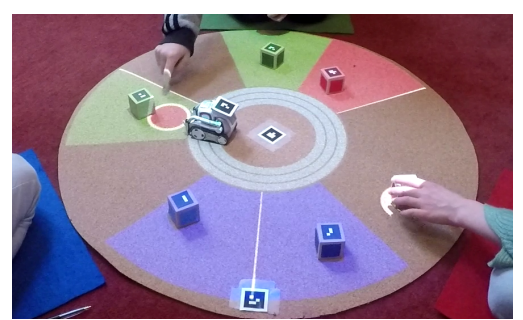

Fig. 2: Overview of the game board, its spatial dimension and the Cozmo robot. The robot joins the activity by suggesting cube placements using its arms to tap at the places of action. Projected lights, here, in the right corner of the picture and in front of Cozmo, help to convey this request.

Robots also have been used as mediators in conflict situations. For example, a robot could promote more constructive conflict solving behavior in case of object possession conflicts among children [13]. When personal violations induce a group conflict, a robot acting as an emotional regulator could help to regulate and call attention to a conflict [9].

Moreover, robots have shown to facilitate collaboration. One work showed that a robot in a moderator role could influence perceived group cohesion by addressing certain participants more often [15]. Another work used a microphone-shaped robot, which could balance the conversation of a group of three and thereby achieve higher group performance [20]. The robot could encourage passive members to participate more actively with non-verbal and indirect cues. When improving human-human collaboration among children, relationreinforcing utterances could enhance the perception of team performance [16]. Similarly, a robot was shown to be able to increase motivation through relationship-building and encouraging behaviors in a Lego-building task [14].

To address inclusion of outgroup members, Sebo et al. [18] explored different strategies involving a robot. Their findings suggest that supportive robot utterances can encourage group members that feel excluded to contribute more.

\section{The MUSIC-BASEd PUZZLE}

Based on teacher feedback and the diverse language background of the children from different countries, we designed a game that could be played without extensive use of language. Instead, we formed the activity around a music-based puzzle. We aimed for creating a task that could be achieved in a collaborative or non-collaborative fashion, such that we can measure the unbiased interaction behavior of heterogeneous groups of children and to create a fair comparison for the study design. The game design was incrementally prototyped through a pilot study in order to create and refine a suitable task for the game and to improve the visual details.

The developed game is a music-based activity played on the ground by three players. A round board ( $\sim 80 \mathrm{~cm}$ diameter) serves as the game area which is divided into three equallysized player regions. Each player is seated in front of one region. This way, all players can reach the different areas on

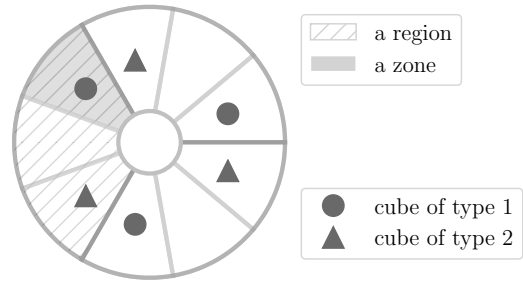

Fig. 3: Exemplary solution configuration for placement of cubes depending on the two types.

the board with similar effort. Each of the three player regions is further divided into three music zones which results in a total of nine different zones. Each player has one region right in front of them, referred to as their own region. The two other regions are more distant for this player and will be referred to as distant regions (see Fig. 2 for an overview on the board).

Cubes are used as tangible elements and can activate the playing of music in different music zones. The system responds with different sound samples in each of the different zones for each cube (in total 54 different samples from 9 music zones and 6 cubes). We further assign the cubes one of two different types to make the puzzle more challenging. We assign a color to each player and hand them two cubes, one of each type. All six cubes can be placed on the board simultaneously. We extracted the sound samples from Incredibo $\sqrt{1}$

Players are instructed to explore the different samples and try to find the nice music mix. The nice music mix - the solution to the puzzle - is thereby a predetermined configuration of placement of cubes that participants have to find. The music samples are therefore no active part of the game but serve as a component that keeps engagement and enjoyment high.

To support the process of finding the puzzle solution, the number of correctly placed cubes is indicated to the players in the middle of the board (see Fig. 2 for an example of three correctly placed cubes). To construct the solution configuration, we define a position for each cube type for one region and replicate this positioning in each region. The correct placement of cubes thereby only depends on the type of the cube and not the color Each cube has to be placed in one of the three different possible positions for its type on the board. Therefore, many different solution combinations are possible. Fig. 3 visualizes one exemplary solution and the respective cube placement.

\section{A. Exploration of music zones}

During the interaction, the system automatically tracks each placement of the cubes of each player. Thereby, the exploration of the different zones can be evaluated online. Exploration of a zone is defined as placement of the cube in that zone and thereby activating the respective sound sample. For each player, we observe a Region Exploration Index (REI) for each region. The $R E I$ encodes the number of different zones of the same region that were explored by each player.

\footnotetext{
${ }^{1}$ https://www.incredibox.com/
} 


\section{B. System implementation}

The game was implemented in Unity and we use a projector and a webcam mounted above the game board. The camera tracks the cubes, the board and the robot. The projector helps to clarify the game and to augment the robot behavior.

\section{ROBOT MEDIATION BEHAVIOR}

The robot's role can be described as a mediator and progress guard. During the game, the robot is showing different behaviors. At predefined intervals, the robot is actively mediating by inviting players to take certain actions, ensuring certain progress during the activity. Between these moments, the robot utilizes indirectly-mediating behaviors.

\section{A. Directly-mediating behavior}

Due to the circular shape of the game board and respective seating of participants, the robot interacts in the center of the activity with equal access to each of the players. The robot joins the interaction by suggesting cube placements by firstly directing the players' attention to the cube targeted for the placement suggestion and then requesting a pick-up. Afterwards, the target place is brought to attention and a put-down is encouraged. To allow for action corrections in case participants are not following these requests, feedback behavior is used to endorse successful cube placement or to disapprove wrong placement or ignorance.

Pilot experiments showed that players mainly move cubes within their own region, which informed the game design and choice of the mediating robot behavior. The robot's goal is to encourage exploration of different placements of the tangible elements for finding the solution to the puzzle. Thereby, the robot does not make a difference between the participants playing the game. The decision to treat all players equally to avoid the robot's attention being only targeted on the outgroup player was supported by results from [15]. Further, we want to ensure that a cohesive group could be formed by encouraging equal participation. To achieve this, the robot utilizes the online evaluation of the cube placements in form of the REI capturing the exploration of the different regions and the progress towards the puzzle solution. Each time the robot intervenes in the interaction, it collects the $R E I_{p r}$ for the players, where index $p \in 0, \ldots, N$ denotes the player (with $N$ players) and index $r \in 0, \ldots, M$ the regions (with $M$ different regions) (in our case, $M=3$ and $N=3$ ). Simultaneously, the progress in the game, i.e., the number of correctly placed elements, is evaluated.

If the solution of the game is progressing as intended, the robot suggests a cube movement that is purely based on the automatically extracted exploration behavior fostering collaboration. Using the information on exploration of regions $R E I$, the player $P_{p}$ and the least explored player region $R_{r}$ that will be the target for the next action are selected:

$$
P_{p}, R_{r} \leftarrow \underset{p, r}{\arg \min }\left\{R E I_{p r}\right\},
$$

for $p \in\{1, \ldots, N\}, r \in\{1, \ldots, M\}$. The robot chooses one cube of this player and suggests a cube placement in the least

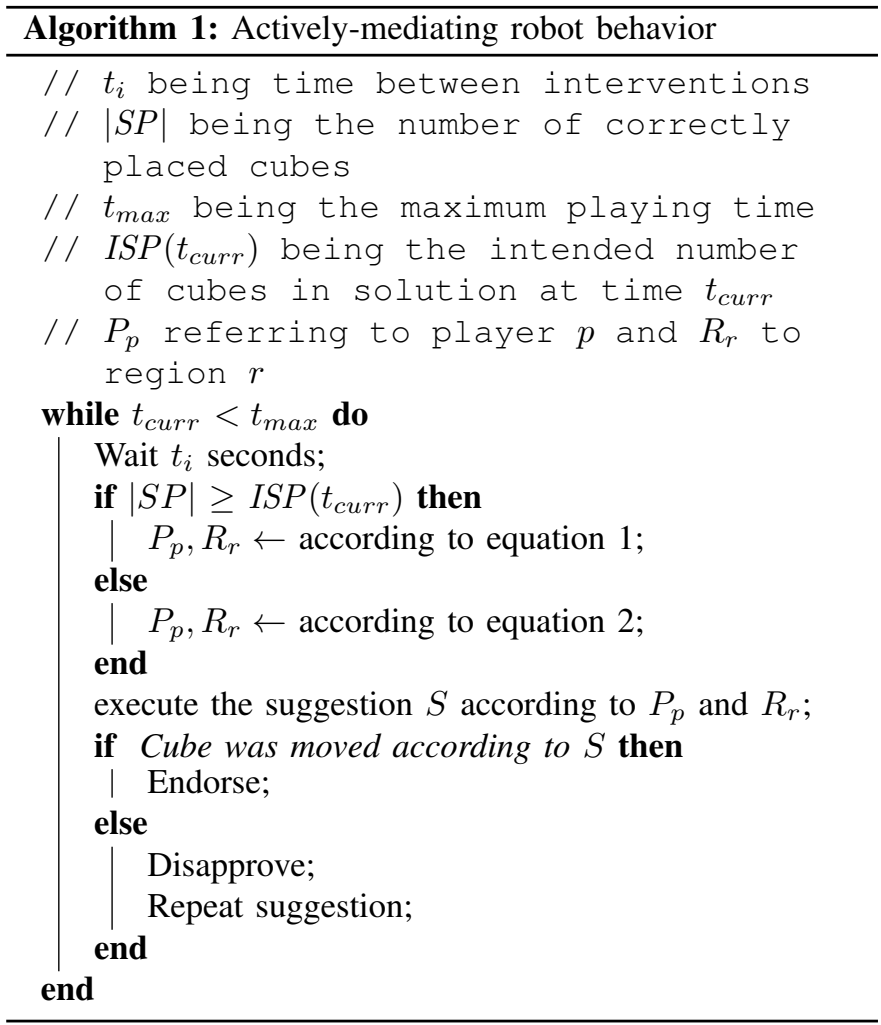

explored player region, preferably in a music zone that has not been explored before.

If the progress in the game at the time of intervention is not as expected, the robot instead suggests a cube movement that helps solving the puzzle. The selection of player $P_{p}$ and region $R_{r}$ then follows:

$$
\begin{aligned}
P_{p}, R_{r} \leftarrow \underset{p, r}{\arg \min }\{ & R E I_{p r} \mid \forall(p, r): \\
& \exists C_{p i} \text { such that } T\left(C_{p i}\right) \in O P\left(R_{r}\right) \\
& \left.C_{p i} \notin S P\right\},
\end{aligned}
$$

for $p \in\{1, \ldots, N\}, r \in\{1, \ldots, M\}$, and $i \in\{1, \ldots, K\}$, where $C_{p i}$ is one of $K$ cubes of player $P_{p}, T(c)$ denotes the type of a cube $c . \operatorname{OP}\left(R_{r}\right)$ describes the set of all open puzzle positions in region $R_{r}$ on the board and $S P$ describes all cubes that are already placed correctly. The cube that needs to be moved to $R_{r}$ to approach the solution of the puzzle is selected for the suggestion. An overview on the algorithm in pseudocode is given in algorithm 1 . Through this actively-mediating behavior selection, the robot encourages the players to explore novel music zones, while ensuring a certain progress in the game when players have difficulties finding the solution.

\section{B. Indirectly-mediating and Idle behavior}

In between the active interventions, the robot shows indirectly-mediating or idle behaviors. Most of the time, it would try to dance to the music. For realizing the indirectlymediating behavior, we use the follow and encourage actions proposed by Tennent et al. [20]. The follow action is realized 
as a reaction to a cube placement targeting the player placing the cube. The encourage action is triggered at predefined time steps $t_{e}$ using equation 1 to decide which player should be targeted.

\section{USER STUDY}

To evaluate the impact of the robot's mediating behavior on children's group participation levels and prosocial behavior, we conducted a between-subject experiment with groups of three children. We formed groups with a pair of children from one classroom and a single child from another classroom. We expect the single child to be perceived as an outgroup member by the pair of ingroup children. To have a more realistic setting of group memberships, the outgroup child in each group is from a classroom of newly arrived children, i.e., children who arrived in Sweden within the last two years.

We designed the experiment around two rounds of the music-based puzzle. In the first game round, the robot interacts with the group of three and influences the children's behavior. To study if this behavior would persist also without the presence of the robot, the second game round is played by the group but without the robot.

\section{A. Hypotheses}

As work by Tennent et al. [20] suggests, encouraging passive or shy players is a feasible robot behavior that could balance the game play. Further, the robot attempts to achieve equal exploration and, as suggested by pilot studies, players will mainly place their cubes right in front of them. Therefore, the robot will focus its attention on encouraging cube placements in increasingly distant zones. By addressing different players, group cohesion could be increased as explored by [15] so that children include the former outgroup child in the ingroup. Based on these findings in prior work and the experimental design, we form the following hypotheses:

1) Hypothesis 1 (H1): Children playing the game with a mediating robot will participate in the puzzle solving task more equally.

2) Hypothesis 2 (H2): Children playing the game with a mediating robot will place their cubes more often outside of their own player region.

3) Hypothesis $3(\mathrm{H3})$ : Ingroup children playing the game with a mediating robot will display more prosocial behavior towards the outgroup members.

\section{B. Conditions}

Each group of three children was randomly assigned to one of the two conditions, the mediation or the control condition. We used the commercially available Cozmo robot which is a palm-sized car-like robot with a pixel face that is used to express emotions. Table I summarizes how Cozmo, supported by augmented reality, executed the different behaviors, i.e., suggesting cube movements, feedback and indirectly-mediating. An example of how Cozmo suggests a cube movement is given in Fig. 2 .

In the mediation condition, cube placement suggestions are generated as described in section IV] In the control condition,

\begin{tabular}{l|l|l} 
Robot behavior & Robot & \multicolumn{2}{|c}{ Our implementation } \\
\hline pick-up & $\begin{array}{l}\text { Cozmo taps its arms close } \\
\text { to the cube. } \\
\text { Cozmo taps its arms on the } \\
\text { game board at the target po- } \\
\text { sition. }\end{array}$ & $\begin{array}{l}\text { White circular } \\
\text { spot on cube. } \\
\text { Pulsing circle } \\
\text { in the color of } \\
\text { the cube. }\end{array}$ \\
\hline feedback & $\begin{array}{l}\text { Happy and sad animations } \\
\text { from Cozmo's SDK. }\end{array}$ & - \\
\hline follow, encour- & $\begin{array}{l}\text { Cozmo turns to player. } \\
\text { age }\end{array}$ & - \\
\hline dance & $\begin{array}{l}\text { Online generation with the } \\
\text { help of a beat detector } \\
{[4] \text { and a rule system for }} \\
\text { Cozmo's movement. }\end{array}$ & -
\end{tabular}

TABLE I: Implementation of the different robot behaviors. We utilized a Cozmo robot and supported its non-verbal behavior by Augmented Reality (AR) elements.

the robot was showing the same behaviors but could not utilize automatically extracted real-time data. Therefore, it was randomly choosing a child and suggested to move a cube with $50 \%$ chance to a zone in front of the child. The other $50 \%$ of the times, the robot would suggest a zone in the regions in front of the other two players. Instead of the follow and encourage behavior, the robot turned randomly to one of the players after 3-10 seconds. As times between interventions, we used $t_{i}=45$ seconds and for encouraging players, we chose $t_{e}=30$ seconds for both mediation and control condition

The robot decision and perception system was supported by a Wizard of Oz setting. The wizard helped the robot to perceive if a child successfully followed its suggestion. Further, the wizard had the possibility to stop the suggestion execution system to keep control for the case of unforeseen errors in the system. All robot behaviors such as suggestion computations and execution, indirectly-mediating and idle behaviors are otherwise fully autonomous.

\section{Measures}

1) Automatic In-Game Measures: As described above, the positions of the cubes and the robot are tracked and we evaluate the REI in real-time. Further, we track each placement of each cube in the zones and regions. We consider a new cube placement as the movement of one cube from one zone to another zone. Measurement errors induced by children not moving their own cubes were corrected through video consultation. To allow further understanding of cube placements, we normalized the cube placements and zone enters to be independent of the seating at the board. In case the participant was from the ingroup, the normalization resulted in the other ingroup player sitting to the right and the outgroup player sitting on the left side of the participant. This allows us to understand if ingroup children made a difference between zones of other ingroup and outgroup players when placing cubes

As a result, we count the total number of placements completed in a game round. Further, we compute zone visit 


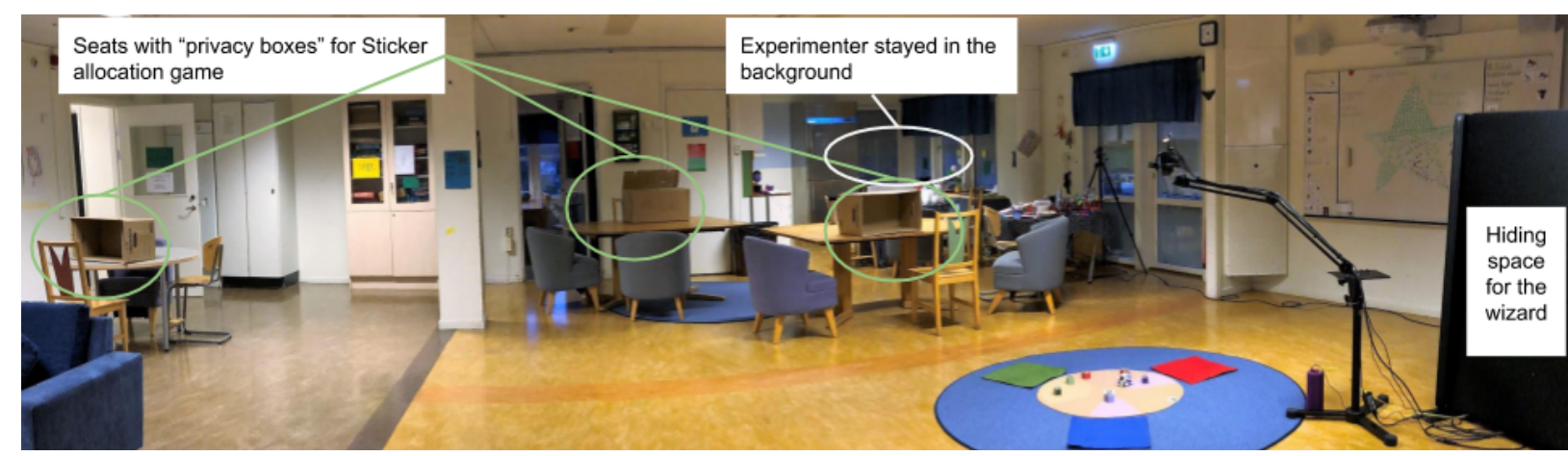

Fig. 4: Experimental space in a separate room of the elementary school.

frequencies for each child and each zone representing how often each of the 9 different zones were visited by the child.

To compare participation within a group, we estimate the variability of participation within each group of three with the coefficient of variation $(C O V)$ of the total count of cube placements of each player in a group. The $C O V$ is computed as $C O V=\frac{s d}{m}$, where $s d$ corresponds to the standard deviation and $m$ to the mean of the total number of placements from the three children in a group.

2) Sticker Allocation: To measure the intuitive display of in- and outgroup-membership, we performed a Dictator Game in form of a sticker allocation task similar to [22, 8]. In a typical dictator game, participants are given an amount of resources and asked to share any number of these resources - stickers in our case. Following previous work, we decided to play the Sticker Allocation Game twice, once allocating stickers to the ingroup classroom and once to the outgroup classroom. In their work, Yu et al. show that children 9 to 10 years old show differences in their sharing behavior between friends and total strangers of the same age [22]. They chose more often the selfish allocation option when allocating stickers to a stranger. Further, these differences in sharing became more apparent when children first allocated their resources to an ingroup member. Therefore, we chose to always start with the allocation to the ingroup members and continue with the allocation to the outgroup members.

As a measure, we counted the allocated stickers to the ingroup and outgroup classroom.

\section{Experimental procedure}

In Sweden, newly arrived children, i.e., those who have lived in the country for less than 2 years, aged 9 and older are firstly taught the Swedish language in a separate language classroom $(L C)$. We expect the children from the $L C$ to be seen as members of an outgroup by the other children who we recruited from other classrooms $(O C)$ of the same school. The study was ethically approved by the Swedish Ethical Review Board (Dnr 2019-05085).

Prior to the study, we obtained the children's guardians' consent. For the $L C$ children, guardians reported the child's previous country of residence and how long the child had been living in Sweden. Children were randomly paired up with similar-aged children where one child always originated from the $L C$ classroom and two children from the $O C$. Each triad was randomly assigned to one of the two conditions (mediation, control). The experiment took place in a separate room at the school and lasted in total $\sim 30$ minutes.

The experimenter first let the children pick a color each and then introduced them to Cozmo and the cubes. To ensure that the children would understand Cozmo's suggestive behavior during the game, children played a warm-up activity with one cube in which Cozmo asked each child to replace the cube twice (one time in distant region). Cozmo was not introduced as having special knowledge, but the experimenter explained that it would be showing what they could do with the cubes on the game board. While children played the game, the experimenter stayed in the background out of children's sight. After each child successfully followed two suggestions by Cozmo, the experimenter came back to explain the game without revealing Cozmo's role. Then, children started playing the first game round with Cozmo interacting in the middle of the game board. Whenever a group of children found the solution of the game within 3 minutes, a second puzzle with a different solution mix was offered to ensure a minimum time of exposure to the robot behavior. After the children successfully found the solution or the maximum game time was reached ( $\sim 10$ minutes), the experimenter returned to the game area and explained that Cozmo got tired from all the dancing and had to sleep, however, the children would play a second game round without Cozmo.

Then, the Sticker Allocation Game started. The experimenter asked the children to take a seat at one of the three "privacy boxes" (similar to [3]). These "privacy boxes" were used to give the children the possibility to allocate their stickers in private and to avoid socially desirable allocations. Further, we utilized envelopes for placing the allocated stickers for the same reason. The experimenter then explained the Sticker Allocation Game from child to child. First, the experimenter gave two envelopes, one for the kept stickers and one for the shared stickers marked by a note. Then, the experimenter gave four stickers to the child and asked them to allocate as many as they wanted to the classroom of the ingroup children by placing the stickers in the respective envelopes.

It was not mentioned that they would play another round of 


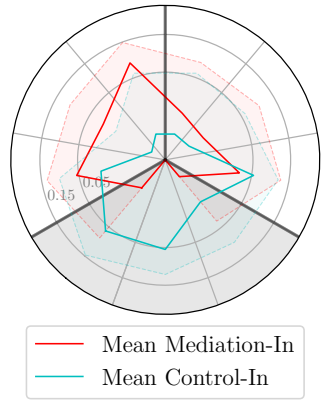

(a) Visualization of robot interventions targeting ingroup players. outgroup children's regions are placed on the left.

Fig. 5: Visualization of frequency of robot interventions in terms of target zones. Color shaded areas symbolize the standard error. The grey shaded area marks the player's own region. Distant regions were left uncolored. The y-axis are scaled by a symmetrical logarithm. Best viewed in color.

allocation game afterwards. When the children were ready, the experimenter removed the envelop with the shared stickers and moved to the second round of the Sticker Allocation Game. Children received a new envelop to allocate the stickers to the classroom of the outgroup children. Instructions for the Sticker Allocation Game, originally from Blake and Rand [3], were translated to Swedish. After allocating the stickers, they were thanked for their participation and asked to return to their classroom.

After all children had completed the session, the experimenter gave envelopes with stickers for distribution in the classroom. The number of stickers in the envelopes were the exact allocations of the children, plus one extra sticker to avoid empty envelopes.

\section{E. Sample}

The participants in this study were fourth and fifth graders of an elementary school that provides an extra language learning classroom to newly arrived children.

Our outgroup participants were recruited from the $L C$. In total, 39 participants, 21 male and 18 female, were recruited for the experiment with a mean age of 10.46 yrs $(S D=.67)$. Out of the 13 groups that participated in the study, three groups were excluded due to a malfunction in the robot or game set-up. Further, two groups found the solution to both puzzles very fast so that the robot did not intervene and were therefore excluded. From the remaining eight groups, four groups interacted in the mediating-robot condition $(M=10.7$ years, $S D=.452$ years, 7 female, 5 male) and four groups in the control condition $(M=10.75$ years, $S D=.622$ years, 4 female, 8 male). The four children (3 female, 1 male) from the $L C$ in the mediation condition have lived in Sweden for an average of 9.5 months $(S D=10.40)$, moving from Asia (1), Europe (2), South America (1). The other four

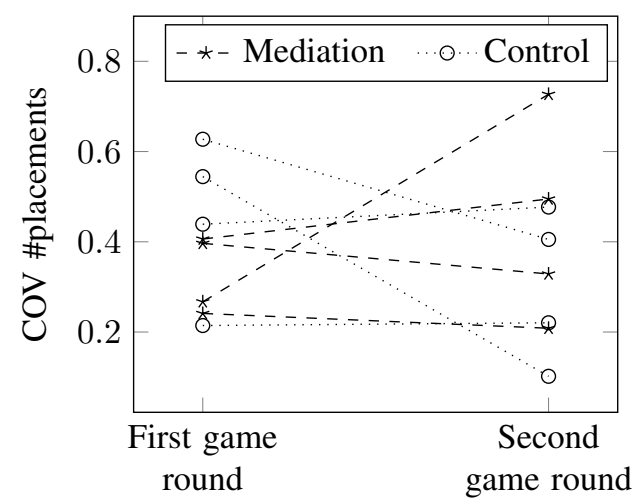

Fig. 6: The $C O V$ describing the variability of the total number of placements in one game round within the groups. Low values of the COV result from low variability in the data indicating a more equal total count of cube placements. Lines connect results from the same group.

children ( 3 female, 1 male) from the $L C$ that participated in the control condition have lived in Sweden for an average of 12.6 months $(S D=10.43)$ and moved from Asia (2), South America (2).

\section{RESULTS}

Given our sample sizes and potential individual characteristics of our outgroup members, we did not conduct a statistical analysis but show trends in the raw values.

\section{A. Manipulation check}

To understand whether the autonomously generated mediation suggestions had the intended outcome, we analysed the interventions in terms of the player and the target zone. The number of interventions addressed at each player were normalized according to the total number of interventions. Fig. 5 displays the frequencies that ingroup and outgroup players were addressed by the robot and where the cube should be placed. Note that the number of outgroup children was by design only half as large as number of ingroup children. For the control condition, the intended manipulation resulted in half the suggestions targeted in the child's own zone and the other half in one of the other two zones for both ingroup and outgroup children.

For both ingroup and outgroup children in the mediation condition, target zones were mostly lying outside of the child's own zone (shaded in grey). This indicates that our assumption that children mainly place cubes in their own regions holds. For ingroup children, most of the interventions were suggesting a cube placement in the outgroup children's region. On average $72.5 \%$ of the suggestions were explorative following equation $1(S D=20.3 \%)$.

\section{B. Equal participation}

To analyse how equally children participated $(H I)$, we used the variability of participation calculated by the $\mathrm{COV}$ of the total count of placements for each group of three. We calculated 


\section{First game round}

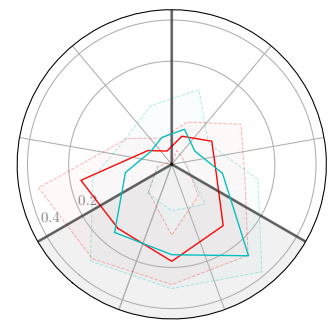

(a) ingroup children's play in the first game round.

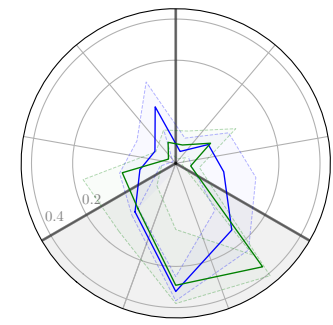

(c) outgroup children's play in the first game round.

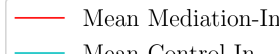

\section{Second game round}

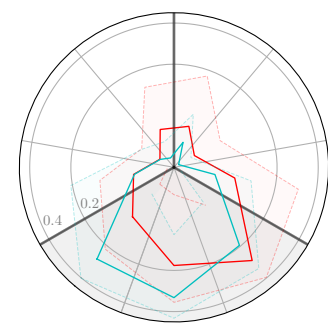

(b) ingroup children's play in the second game round.

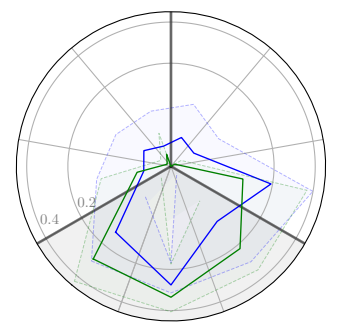

(d) outgroup children's play in the second game round.

_ Mean Mediation-Out

Fig. 7: Zone enter frequencies for the 9 zones on the game board for in- and outgroup children (row-wise) between conditions (within figures) for the first and the second game round (column-wise). Color shaded areas mark the standard error. The grey shaded area marks a player's own region. Distant regions were left uncolored. outgroup children's regions are placed on the left (for ingroup players). The y-axis are scaled by a symmetrical logarithm. Best viewed in color.

this $C O V$ for each game round. Fig. 6 shows the results where high numbers mean high variability in participation, low values resulting from low variability - more equal participation. Considering the first game round, the variability is lower for groups in the mediation condition $(M=0.32, S D=0.07)$ than in the control condition $(M=0.45, S D=0.15)$. This indicates that the game participation was more equal between the children in the mediating condition in the first game round. For the second game round, the variability of participation does not show a clear trend but on average the variability is lower for the groups in the control $(M=0.3, S D=0.14)$ than the mediation condition $(M=0.44, S D=0.19)$.

\section{Exploration of distant zones}

To evaluate $H 2$, we analysed the exploration of distant zones by evaluating the zone visit frequencies which encapsulate the frequency with which cubes were placed in the different zones of the game board. The four figures in Fig. 7 show a preference for placing cubes in the children's own zones (grey shaded

\begin{tabular}{c|c|c} 
& \multicolumn{2}{|c}{ Condition } \\
& Mediation & Control \\
\hline Less & 1 & 1 \\
Equal & 4 & 6 \\
More & 3 & 1
\end{tabular}

TABLE II: Sticker allocation behavior of ingroup participants. The table describes how many ingroup children allocated less/equal/more stickers to the outgroup than to the ingroup classrooms.

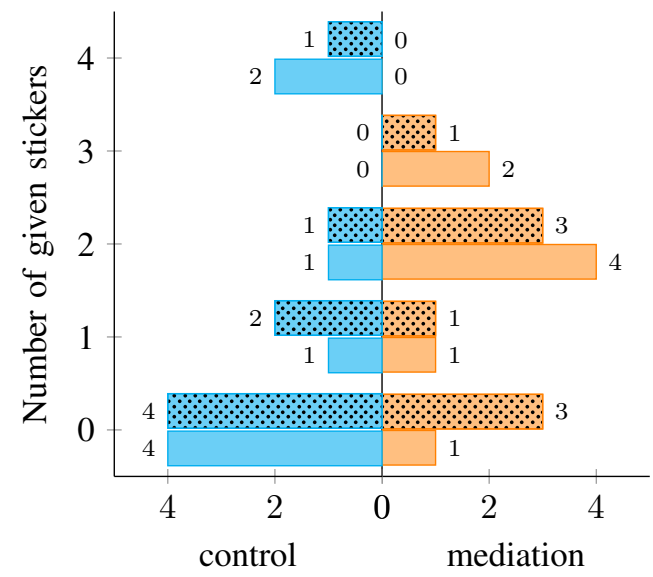

Fig. 8: Count of number of allocated stickers for control (left) and mediation (right) condition given by the ingroup children in the Sticker Allocation Game. Each child had a total of 4 stickers for each of the two allocation tasks (allocation to ingroup (dotted bars) and outgroup (empty bars) classroom).

area) regardless of in- or outgroup membership or condition. Figures $7 \mathrm{a}$ and $7 \mathrm{c}$ show the results of exploration for the first game round. Results for the second game round are found in $7 \mathrm{~b}$ and $7 \mathrm{~d}$. For both in- and outgroup children, the mediation condition shows a trend to more frequent placement of cubes in the 6 distant zones in the second game round. 4 (see Fig. 7b) and 5 (see Fig. 7d) out of the 6 distant zones, for in- and outgroup children respectively, were visited more frequently in the second game round.

\section{Allocation of stickers}

To evaluate $H 3$, we analysed the result of the Sticker Allocation Game. As the children from the $L C$ are in the process of learning Swedish, we could not ensure that each of the children understood the task correctly. Therefore, we only considered the data of the 16 ingroup children, i.e., participants from the $O C$ for the analysis. Table $\mathrm{II}$ shows the difference that the children made between allocating to the ingroup and to the outgroup. Most of the children did not make a difference if they were allocating stickers $(10 / 16)$ to the ingroup or to the outgroup classroom. The frequencies of sticker allocations are depicted in Fig. 8, Overall, children in the mediation condition show a trend to allocate more stickers $(M=1.56, S D=1.09)$ and share them more equally, i.e., giving 2 stickers, than children in the control condition $(M=1.18, S D=1.56)$. 


\section{DISCUSSION}

This study was designed to explore how newly arrived children could be included in a new classroom environment with the help of a robot that fosters group collaboration. We built a robot-involved music-based puzzle as an activity to be played by two ingroup members and one outgroup member. Each group played two game rounds, the first in the presence of the robot and the second game round without the robot.

We hypothesised that more equal participation would be a result of the robot mediating the activity (HI). Results show a trend towards the robot being able to achieve more equal participation during its presence in the first game round. However, the groups in the control condition improved in their second game round and participated more equally. The reasons for this could be manifold. For example, it could be an effect of the robot also suggesting cube placements in distant zones in the control condition. Understanding the underlying causes that lead to more equal participation in the control condition might give valuable insights and encourages future work on how mediation behavior could be designed.

When considering the exact placements of cubes and evaluating $H 2$, the robot's manipulation was successful given that outgroup and ingroup children in the mediating condition, especially in the second game round, placed their cubes more often in the distant zones. However, during the first game round, children from the control condition showed only a small difference in behavior compared to their counterparts in the mediation condition. As the difference is more apparent in the second game round (when the robot is not present), these results indicate that the effect of the robot's interventions persists in the mediating condition.

The findings from $H 1$ and $H 2$ suggest that the robot's mediating behavior had an important role in the inclusion process of the outgroup member. From the perspective of the outgroup member, we observe more frequent visits to distant zones (of the ingroup players), and these frequencies are similar to the patterns that ingroup children show. This indicates that the outgroup children were not shy to get accommodated to the ingroup children. From the perspective of the ingroup children, we observed that cube placements were more often in the zones of the other ingroup child compared to the control condition, especially in the second game round. Going back to the analysis of the robot's suggestions, these were more often targeted at placement towards the outgroup child, which indicates that the robot successfully perceived the group unbalance and actively worked to mediate it. The robot attempts seem successful as ingroup children show a trend towards equal treatment between ingroup and outgroup in regards of cube placements.

In summary, the outgroup children took a first step towards interacting with the ingroup children, and ingroup children took a first step towards including the outgroup child but did not treat the outgroup child fully equal yet.

The shortness of the interaction might explain this result. A repeated interaction experiment spanning over multiple days would be needed to verify these trends, because it may take more than a single intervention to fully include an outgroup child in the ingroup.

Nevertheless, this analysis also shows that the robot's mediation behavior lead to increased collaboration between the two children of the ingroup, resulting in more frequent zone visits in the other ingroup child's region especially in comparison with the control condition.

H3 hypothesised that children are more prosocial towards the outgroup members can be supported. Children in the mediation condition were not only more prosocial towards the outgroup member, but also towards ingroup (indicated by more generous sticker allocations). A known interplay between prosociality and empathic concern [6] offers a potential explanation for the increased prosocial behavior in the mediation condition, and encourages future studies on robotic mediators.

\section{A. Limitations}

Limiting to our study and findings is the small sample size and individual background of our participants that did not allow us to conduct a statistical analysis and show results in terms of significant findings. We studied the influence of personality with the Social Skills Rating System (cooperation, assertion and self-control) filled by the main teachers. Visual inspection did not reveal an effect and cannot be completely excluded without significant indicators. Additionally, we did not measure prior willingness to collaborate within the group of three, which could have influenced our measures. To the best of our knowledge, there is though no validated measure that would allow for evaluation of prior willingness to collaborate that does not rely extensively on the use of language, and therefore would be unsuitable for our target group. Further, we did not study the possible influence of the chosen music samples. Choosing music samples that show the diversity of culture in the group could have had further positive impact on the inclusion of the newly arrived children.

\section{CONCLUSION}

We developed a social robot mediator that aimed at including outgroup members in a music-based puzzle, specifically targeting children newly arrived to a country. The robot's mediating efforts persisted even when children played a game without the robot. The robot succeeded in encouraging the outgroup child to take a step towards the ingroup, and it could increase collaboration between the ingroup members. Despite the fact that ingroup children did not treat the ingroup child fully equal yet, children were more prosocial when the robot mediated the game. As inclusion problems increase with diversity and globalism, the use of assistive robots to overcome these issues, especially among children, seems a promising approach based on our findings.

\section{ACKNOWLEDGMENTS}

This work was partially funded by a Fellowship from the Jacobs Foundation (nr. 20171261 06). We thank Gaspar I. Melsión for assisting in running the study. 


\section{REFERENCES}

[1] World Migration Report 2020. IOM World Migration Report. UN, $112019 . \quad$ ISBN 9789290687894. URL https://www.un-ilibrary.org/migration/ world-migration-report-2020_b1710e30-en.

[2] Frances Aboud. The Formation of In-Group Favoritism and Out-Group Prejudice in Young Children: Are They Distinct Attitudes? Developmental psychology, 39:4860, 022003.

[3] Peter R. Blake and David G. Rand. Currency value moderates equity preference among young children Evolution and Human Behavior, 31(3):210-218, 2010.

[4] Sebastian Böck, Filip Korzeniowski, Jan Schlüter, Florian Krebs, and Gerhard Widmer. madmom: a new Python Audio and Music Signal Processing Library 2016.

[5] Marilynn B Brewer. In-group bias in the minimal intergroup situation: A cognitive-motivational analysis. Psychological bulletin, 86(2):307, 1979.

[6] Jolien der Graaff, Gustavo Carlo, Elisabetta Crocetti, Hans M Koot, and Susan Branje. Prosocial Behavior in Adolescence: Gender Differences in Development and Links with Empathy Journal of Youth and Adolescence, 47(5):1086-1099, 52018.

[7] John Gottman, Jonni Gonso, and Brian Rasmussen. Social interaction, social competence, and friendship in children. Child development, pages 709-718, 1975.

[8] Berna Güroglu, Wouter van den Bos, and Eveline A. Crone. Sharing and giving across adolescence: An experimental study examining the development of prosocial behavior. Frontiers in Psychology, 5(APR):1-13, 2014.

[9] Malte F. Jung, Nikolas Martelaro, and Pamela J. Hinds. Using Robots to Moderate Team Conflict: The Case of Repairing Violations. In Proceedings of the Tenth Annual ACM/IEEE International Conference on HumanRobot Interaction, page 229-236, Portland, Oregon, USA, 2015. Association for Computing Machinery.

[10] Sebastian Kirschner and Michael Tomasello. Joint music making promotes prosocial behavior in 4-year-old children Evolution and Human Behavior, 31(5):354-364, 2010.

[11] Patrick J. Leman. How do groups work? Age differences in performance and the social outcomes of peer collaboration Cognitive Science, 39(4):804-820, 2015.

[12] Bilge Mutlu, Toshiyuki Shiwa, Takayuki Kanda, Hiroshi Ishiguro, and Norihiro Hagita. Footing in human-robot conversations: how robots might shape participant roles using gaze cues. In Proceedings of the 4th ACM/IEEE international conference on Human robot interaction, pages 61-68. ACM, 2009.

[13] Solace Shen, Petr Slovak, and Malte F. Jung. " Stop . I See a Conflict Happening ." A Robot Mediator for Young Children 's Interpersonal Conflict Resolution In Human Robot Interaction, pages 69-77, 2018.

[14] Michihiro Shimada, Takayuki Kanda, and Satoshi Koizumi. How Can a Social Robot Facilitate Children's
Collaboration? In Shuzhi Sam Ge, Oussama Khatib, John-John Cabibihan, Reid Simmons, and Mary-Anne Williams, editors, Social Robotics, pages 98-107, Berlin, Heidelberg, 2012. Springer Berlin Heidelberg.

[15] Elaine Short and Maja J. Mataric. Robot moderation of a collaborative game: Towards socially assistive robotics in group interactions. In RO-MAN 2017 - 26th IEEE International Symposium on Robot and Human Interactive Communication, 2017.

[16] Sarah Strohkorb, Ethan Fukuto, Natalie Warren, Charles Taylor, Bobby Berry, and Brian Scassellati. Improving human-human collaboration between children with a social robot 25th IEEE International Symposium on Robot and Human Interactive Communication, RO-MAN 2016, pages 551-556, 2016.

[17] Sarah Strohkorb Sebo, Margaret Traeger, Malte F. Jung, and Brian Scassellati. The Ripple Effects of Vulnerability: The Effects of a Robot's Vulnerable Behavior on Trust in Human-Robot Teams. Proceedings of the 2018 ACM/IEEE International Conference on Human-Robot Interaction - HRI '18, (February):178-186, 2018.

[18] Sarah Strohkorb Sebo, Ling Liang Dong, Nicholas Chang, and Brian Scassellati. Strategies for the Inclusion of Human Members within Human-Robot Teams pages 309-317, 32020.

[19] Henri Tajfel, Michael G Billig, Robert P Bundy, and Claude Flament. Social categorization and intergroup behaviour. European journal of social psychology, 1(2): 149-178, 1971.

[20] Hamish Tennent, Solace Shen, and Malte Jung. Micbot: A Peripheral Robotic Object to Shape Conversational Dynamics and Team Performance. ACM/IEEE International Conference on Human-Robot Interaction, pages 133-142, 2019.

[21] Dina Utami and Timothy Bickmore. Collaborative User Responses in Multiparty Interaction with a Couples Counselor Robot. In 2019 14th ACM/IEEE International Conference on Human-Robot Interaction (HRI), pages 294-303, 2019.

[22] Jing Yu, Liqi Zhu, and Alan M. Leslie. Children's Sharing Behavior in Mini-Dictator Games: The Role of In-Group Favoritism and Theory of Mind Child Development, 87(6):1747-1757, 2016. 